\title{
Mild therapeutic hypothermia after out-of-hospital cardiac arrest: What does really matter?
}

\author{
Jakub Ratajczak, Piotr Łach, Julia M. Umińska, Krzysztof Pstrąowski, \\ Michał Kasprzak, Tomasz Fabiszak, Eliano Pio Navarese, Jacek Kubica \\ Department of Cardiology and Internal Medicine, Nicolaus Copernicus University, \\ Collegium Medicum, Bydgoszcz, Poland
}

\begin{abstract}
Background: Mild therapeutic hypothermia (MTH) is a recommended treatment of comatose patients after out-of-hospital cardiac arrest (OHCA). The aim of the study was to examine determinants of clinical outcome in OHCA survivors treated with MTH and variables associated with MTH induction time. Methods: Presented herein is an analysis of combined results from a retrospective and a prospective observational study which included 90 OHCA survivors treated with MTH from January 2010 to March 2018. Multivariate regression analysis was performed to determine variables associated with poor neurologic outcome (Cerebral Performance Category 3-5), mortality, and prolonged induction time. Results: At hospital discharge, 59 (65.6\%) patients were alive, of whom 36 (61\%) had a good neurologic outcome. Older patients (odds ratio [OR] 1.07, 95\% confidence interval [CI] 1.03-1.12) with lower Glasgow Coma Scale (GCS) (OR 0.49, 95\% CI 0.30-0.80) were at higher risk of poor neurological outcome. The predictors of in-hospital death included: older age (OR 1.08, 95\% CI 1.02-1.13), lower GCS score (OR 0.47, 95\% CI 0.25-0.85), presence of cardiogenic shock (OR 3.43, 95\% CI 1.11-10.53), and higher doses of adrenaline (OR 1.27, 95\% CI 1.04-1.56). Longer induction was associated with shorter cardio-pulmonary resuscitation (CPR) (unstandardized coefficient $-3.95,95 \%$ CI-7.09 to -0.81) and lower lactate level (unstandardized coefficient-18.55, 95\% CI-36.10 to-1.01).

Conclusions: Unfavorable neurologic outcome in OHCA patients treated with MTH is associated with age and lower GCS score. Risk factors for in-hospital mortality include age, high-dose adrenaline administration, lower GCS score and presence of cardiogenic shock. CPR duration and lactate level were predictive of prolonged MTH induction time. (Cardiol J XXXX; XX, X: $\mathrm{xX}-\mathrm{Xx}$ )

Key words: mild therapeutic hypothermia, targeted temperature management, out-of-hospital cardiac arrest
\end{abstract}

\section{Introduction}

Out-of-hospital cardiac arrest (OHCA) is still burdened with high risk of death. Among those who experience OHCA only around $30 \%$ survive before hospital admission [1] and $10 \%$ until hospital discharge [2]. The risk of OHCA occurrence is greater among the population of older men [3]. Even in survivors, brain damage leads to impaired neurological function and their ability to live an independent life is often limited [4]. Current guidelines recommend targeted temperature management (TTM) for the treatment of comatose patients after OHCA [5, 6], however the results presented in the literature regarding cardiac arrest patients with non-shakable rhythms are inconclusive [7]. TTM is a term most commonly understood as maintenance of the body's core temperature between $32^{\circ} \mathrm{C}$ and $36^{\circ} \mathrm{C}$ and therefore covers a wider range than mild therapeutic hypothermia (MTH),

Address for correspondence: Jakub Ratajczak, MD, Department of Cardiology and Internal Medicine, Nicolaus Copernicus University, Collegium Medicum in Bydgoszcz, ul. Marii Skłodowskiej-Curie 9, 85-094 Bydgoszcz, Poland, e-mail: ratajczak.j.m@gmail.com

Received: 10.01.2019 Accepted: 17.02.2019 
defined as temperature control between $32^{\circ} \mathrm{C}$ and $34^{\circ} \mathrm{C}$. While favorable clinical outcome of MTH was proven in several clinical studies [8-10], the impact of maintaining patient temperatures at $34-36^{\circ} \mathrm{C}$ remains less clear. A randomized study by Nielsen et al. [11] suggests equivalent results in patients treated with TTM at $33^{\circ} \mathrm{C}$ and $36^{\circ} \mathrm{C}$. Significant variability in clinical outcome among subjects treated with TTM raises questions regarding determinants of treatment success [12]. Despite the use of pre-specified programmed pattern of cooling, several studies reported that patients with poor neurological outcome had significantly shorter time required to achieve the target temperature (TT) than patients with good outcome [13-15]. These results raises the question about the causes of this phenomenon. Herein is hypothesized that a more severe ischemic insult may result in greater brain damage leading to impaired thermoregulatory control. According to this assumption, individuals with better post-OHCA neurologic function preserve their thermoregulation ability, which in turn results in longer induction time of MTH. However, the question as to whether length of MTH induction is an indicator of brain damage severity and what are the predictors associated with prolonged MTH induction remains unanswered.

Therefore, in this study possible determinants of clinical outcome in OHCA survivors treated with MTH were examined, including the induction time. Variables associated with the duration of MTH induction were also evaluated.

\section{Methods}

\section{Study design and TTM protocol}

This study combines results of a retrospective observational single-center analysis performed at the Department of Cardiology and Internal Medicine of the University Hospital No. 1 in Bydgoszcz, Poland from January 2010 to December 2016 and a prospective, observational, multicenter study [16] performed from January 2017 to March 2018 including OHCA survivors treated with MTH. The study comprised all consecutive adult subjects treated with MTH (using invasive intravascular cooling with $\mathrm{TT}$ of $33^{\circ} \mathrm{C}$ ) for non-traumatic $\mathrm{OHCA}$ regardless of initial rhythm, who achieved a return of spontaneous circulation (ROSC). The Utsteinstyle guidelines for reporting OHCA were implemented in the study [17].

According to local protocol, patients were considered for MTH if they remained comatose after return of ROSC. Cooling was initiated as soon as possible with ice packs, intravenous administration of cold normal saline $(0.9 \%$ solution of sodium chloride at the temperature of $4^{\circ} \mathrm{C}$ ), and Intravascular Temperature Management ${ }^{\mathrm{TM}}$ CoolGard $3000^{\circledR}$ (Zoll Circulation Inc. USA). MTH was considered effective when patient core temperature decreased below $34^{\circ} \mathrm{C}$, with TT of $33.0 \pm 0.2^{\circ} \mathrm{C}$, and was maintained for at least $12 \mathrm{~h}$ with an optimal duration of $24 \mathrm{~h}$. The rewarming phase was conducted in an actively controlled manner at a rate of $0.3^{\circ} \mathrm{C}$ per hour. Urine bladder temperature measurements were used to automatically guide changes in patient core temperature. All patients were mechanically ventilated, sedated with continuous intravenous infusion of propofol and fentanyl and were treated according to current European Society of Cardiology guidelines. More detailed information regarding MTH protocol has been previously described [10].

The study was conducted in accordance with the Declaration of Helsinki and Good Clinical Practice guidelines and was approved by the Ethics Committee of The Nicolaus Copernicus University in Torun, Collegium Medicum in Bydgoszcz (approval reference number KB 615/2015). The prospective part of this study is a sub-study of the Mild Therapeutic Hypothermia for Patients With Acute Coronary Syndrome and Cardiac Arrest Treated With Percutaneous Coronary Intervention (UNICORN) study (ClinicalTrials.gov Identifier: NCT02611934), which was supported by "Diamentowy Grant" financed by the Ministry of Science and Higher Education of the Republic of Poland from research funds for the years 2015-2018.

\section{Data collection}

Data were obtained from hospital records and included: age, sex, comorbidities, first monitored rhythm, bystander basic life support (BLS), total dose of adrenaline (epinephrine) used by emergency medical service (EMS) during cardiopulmonary resuscitation (CPR), etiology of cardiac arrest, Glasgow Coma Scale (GCS) score on admission, blood $\mathrm{pH}$ on admission, blood lactate level on admission, left ventricular ejection fraction on admission, presence of cardiogenic shock, and initial temperature measured in the urinary bladder (recorded at the initiation of intravascular cooling). Time intervals used for the analysis included: time in cardiac arrest (time from the onset of OHCA to ROSC), CPR duration (time from the beginning of CPR by EMS to ROSC), pre-induction (time from ROSC to initiation of intravascular cooling), induction (time from initiation of intravascular cooling to arrival at TT of $33.0 \pm 0.2^{\circ} \mathrm{C}$ ), maintenance (time 




Figure 1. Numbers of patients initially screened, those excluded from the study and finally those who were included for analysis; GCS - Glasgow Coma Scale; IHCA — in-hospital cardiac arrest; MTH — mild therapeutic hypothermia.

from arrival at TT to initiation of active rewarming). Neurologic outcome was assessed using the Glasgow-Pittsburgh Cerebral Performance Categories (CPC) at hospital discharge and recorded as CPC 1 (good performance), CPC 2 (moderate disability), CPC 3 (severe disability), CPC 4 (vegetative state), or CPC 5 (brain death or death) [18]. Good neurological outcome was defined as CPC 1-2 and poor neurologic outcome was CPC $3-5$ at the time of hospital discharge.

\section{Statistical analysis}

Continuous variables were presented as means with standard deviation or medians with interquartile range according to the distribution. Categorical variables were described as frequencies and percentages. Normality of the distribution was examined with the Shapiro-Wilk test. The Student T-test or Mann-Whitney test were used for comparison of continuous variables based on distribution normality. Categorical variables were compared using the $\chi^{2}$ or Fisher's exact test, as appropriate. Univariate logistic regression analysis was performed to determine variables associated with poor neurologic outcome and in-hospital mortality. In order to identify variables affecting MTH induction time, univariate linear regression analysis was conducted. All variables significant at $\mathrm{p} \leq 0.1$ in the univariate analysis were entered into multivariate regression models. Stepwise regression with backward elimination was performed to find the best possible fitting of each model. All statistical analysis was performed with IBM SPSS Statistics version 23. A two-sided significance level of $\mathrm{p}<0.05$ was applied for statistical significance.

\section{Results}

\section{Population characteristics} and final outcome

A total of 114 adult cardiac arrest patients were treated with MTH during the study period. Twenty-four patients were excluded from analysis (Fig. 1) and data on the remaining 90 patients were further investigated. At hospital discharge, 59 $(65.56 \%)$ patients were alive, of whom $36(61.02 \%)$ had a good neurologic outcome. Their clinical characteristics stratified by outcomes is shown in Table 1 . The study group consisted mainly of men $(\mathrm{n}=72,80 \%)$. Mean age was $61.8 \pm 12.5$ years. In the majority of patients $(\mathrm{n}=79,87.8 \%)$, the initial recorded rhythm was shockable. Patients with good neurologic outcome were younger than those with poor outcome $(56.4 \pm 12.1$ vs. $65.3 \pm$ $11.5, \mathrm{p}=0.001)$, had a higher incidence of shockable initial rhythm (100\% vs. $79.6 \%, p=0.003)$ and a higher GCS score on admission (4.0 [4.0-5.0] vs. 3.5 [3.0-4.0], $\mathrm{p}=0.004)$.

Survivors, as compared with non-survivors, were younger $(59.3 \pm 12.1$ vs. $66.5 \pm 12.0$, $\mathrm{p}=0.009)$, more likely to have shockable initial rhythm (93.2\% vs. $77.4 \%, \mathrm{p}=0.04)$ and less likely to present with cardiogenic shock ( $45.8 \%$ vs. $71.0 \%$, $\mathrm{p}=0.02)$. They also required smaller amounts of adrenaline during CPR (2.0 mg [1.0-5.0] vs. $4.0 \mathrm{mg}$ [2.75-7.0], $\mathrm{p}=0.006)$ and had a higher GCS score on admission (4.0 [3.0-5.0] vs. 3.0 [3.0-4.0], $\mathrm{p}=0.004]$ (Table 1$)$.

According to the univariate analysis, lower admission GCS score, older age and shorter MTH induction time were associated with poor neuro- 


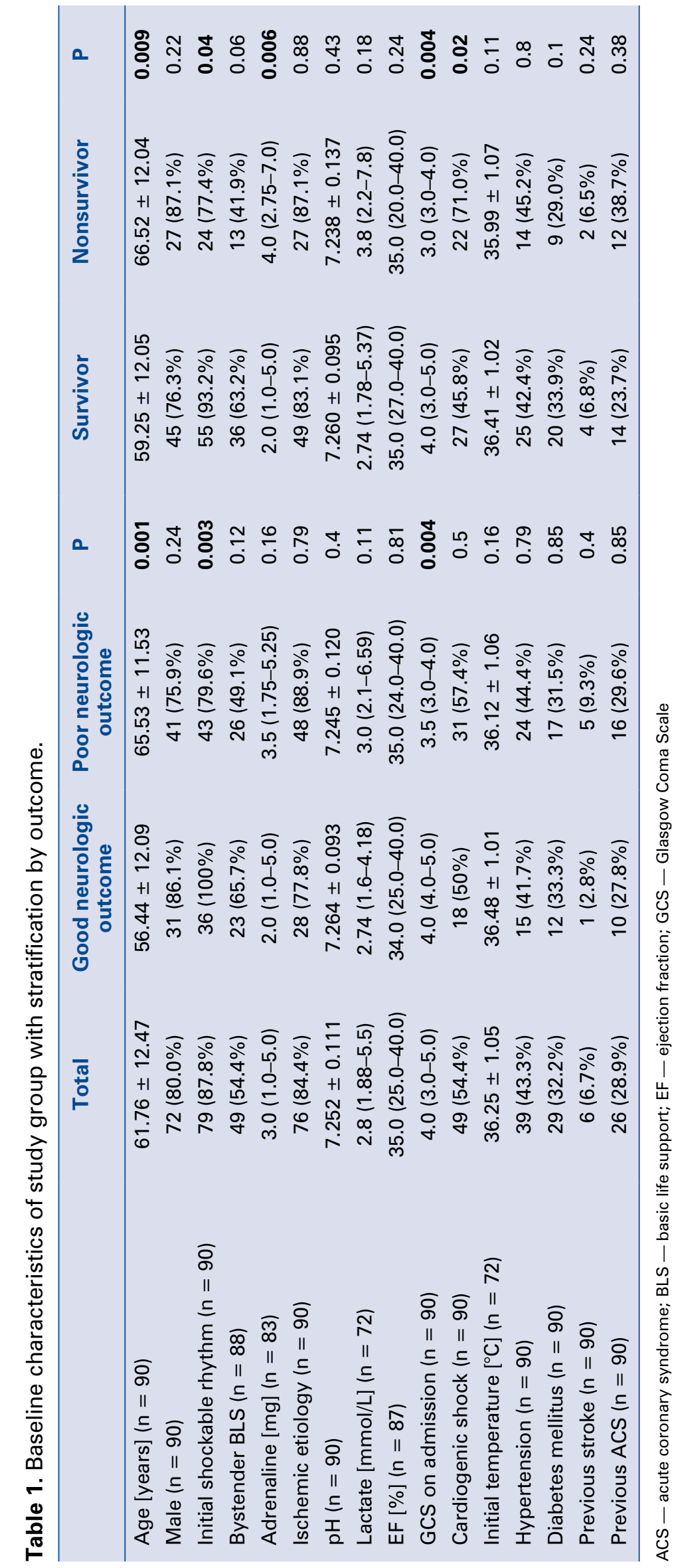


logical outcome (CPC 3-5) at hospital discharge (Table 2). As a result of stepwise regression with backward elimination, two parameters lactate level and MTH induction time - were excluded from the equation. The best fitted multiple regression model revealed that older patients (odds ratio [OR] 1.07, 95\% confidence interval [CI] 1.03-1.12, $\mathrm{p}=0.001$ ) and those with a lower GCS score on admission (OR 0.49, 95\% CI $0.30-0.80, \mathrm{p}=0.004)$ were at higher risk of poor neurological outcome.

Analogous analysis was conducted to determine risk factors of non-survival. In the univariate analysis, older age, higher adrenaline dosage during CPR, lower admission GCS score and presence of cardiogenic shock were associated with higher in-hospital mortality (Table 2). For multivariate analysis two new variables were added, i.e. CPR duration and bystander BLS. Eventually, stepwise regression revealed older age (OR $1.08,95 \%$ CI $1.02-1.13, \mathrm{p}=0.006$ ), lower GCS score (OR 0.47, $95 \%$ CI $0.25-0.85, \mathrm{p}=0.01)$, presence of cardiogenic shock (OR 3.43, 95\% CI 1.11-10.53, p = 0.03), and higher doses of adrenaline during CPR (OR 1.27, 95\% CI 1.04-1.56, $\mathrm{p}=0.02$ ) to be risk factors of in-hospital death.

\section{Determinants of the duration of MTH induction}

Patients who recovered with a CPC of 1 or 2 , had a significantly longer median induction time than patients with a CPC of $3-5$ (325.0 $\mathrm{min}$ [230.0-615.0] vs. $260.0 \min$ [180.0-360.0], $\mathrm{p}=0.04$; Table 3 ). According to univariate analysis, lower lactate level, higher GCS score on admission, higher initial body temperature, shorter time in cardiac arrest and shorter CPR duration were significantly associated with the duration of MTH induction (Table 4). As a potential confounder, initial temperature was excluded from further analysis. According to multivariate analysis, longer duration of MTH induction was independently associated with shorter CPR duration [unstandardized coefficient $-3.95,95 \% \mathrm{CI}$ -7.09 to $-0.81, \mathrm{p}=0.01$ ) and lower lactate level (unstandardized coefficient $-18.55,95 \%$ CI -36.10 to $-1.01, \mathrm{p}=0.04$ ).

\section{Discussion}

No differences were found regarding MTH induction time in terms of OHCA survival, however patients with poor neurologic outcome had significantly shorter MTH induction time than patients with a good outcome. Older age and lower GCS score on admission were also identified as independent predictors of worse neurologic outcome as well as older age, higher adrenaline dosage during CPR, lower baseline GCS score and presence of cardiogenic shock as independent risk factors of mortality. CPR duration and lactate concentration were independently associated with the duration of MTH induction.

Previous studies delivered inconsistent results regarding the relationship between MTH induction time and neurologic outcome. Nielsen et al. [19] reported that neither time to initiation of TTM, time to achieve TT, duration of TTM nor rewarming time were associated with neurologic outcome. An analysis of 588 patients conducted by Haugk et al. [13] indicated that patients with favorable outcome had both longer - time from ROSC to TT as well as induction time, with no difference in time from ROSC to initiation of cooling. They also performed a multivariate regression analysis and found $86 \%$ higher odds of a good neurologic outcome with an increase in each tertile ( $<120 \mathrm{~min}, 120-220 \mathrm{~min}$, and $>220 \mathrm{~min}$ ) of time to TT (adjusted OR 1.86, $\mathrm{p}=0.04$ ). The median time needed to achieve TT, despite numerical difference, was not associated with overall survival (202 min for survivors vs. 158 min for non-survivors, $\mathrm{p}=0.57$ ). Several procedure-related differences between the studies and within particular studies should be underlined. In the study by Haugk et al. [13], TT was defined as less than $34^{\circ} \mathrm{C}$ and different cooling methods including endovascular, head cooling, surface (ice, water, air), nasopharyngeal, intravenous, and mixed were applied. Perman et al. [14] analyzed 321 patients from various centers and categorized them by induction time (<120 min, $120-300 \mathrm{~min},>300 \mathrm{~min}$ ). The authors reported that age, shockable initial rhythm, time in cardiac arrest and induction time $>300$ min were associated with a higher probability of a favorable neurologic outcome. In contrast to this, other publications showed rapid TT achievement to be associated with better neurologic results [20-23]. However, all those studies defined time to TT as the period from the onset of cardiac arrest to arrival at a temperature of $33^{\circ} \mathrm{C}$ or $<34^{\circ} \mathrm{C}$, therefore it was equivalent to three different time intervals used in the current study (time in cardiac arrest, pre-induction and induction). In each of these time periods patients are affected by various conditions potentially determining outcome, thus we believe that the analysis should be conducted separately for each of these time intervals. Shorter time in cardiac arrest $[19,24]$ and sooner initiation of 

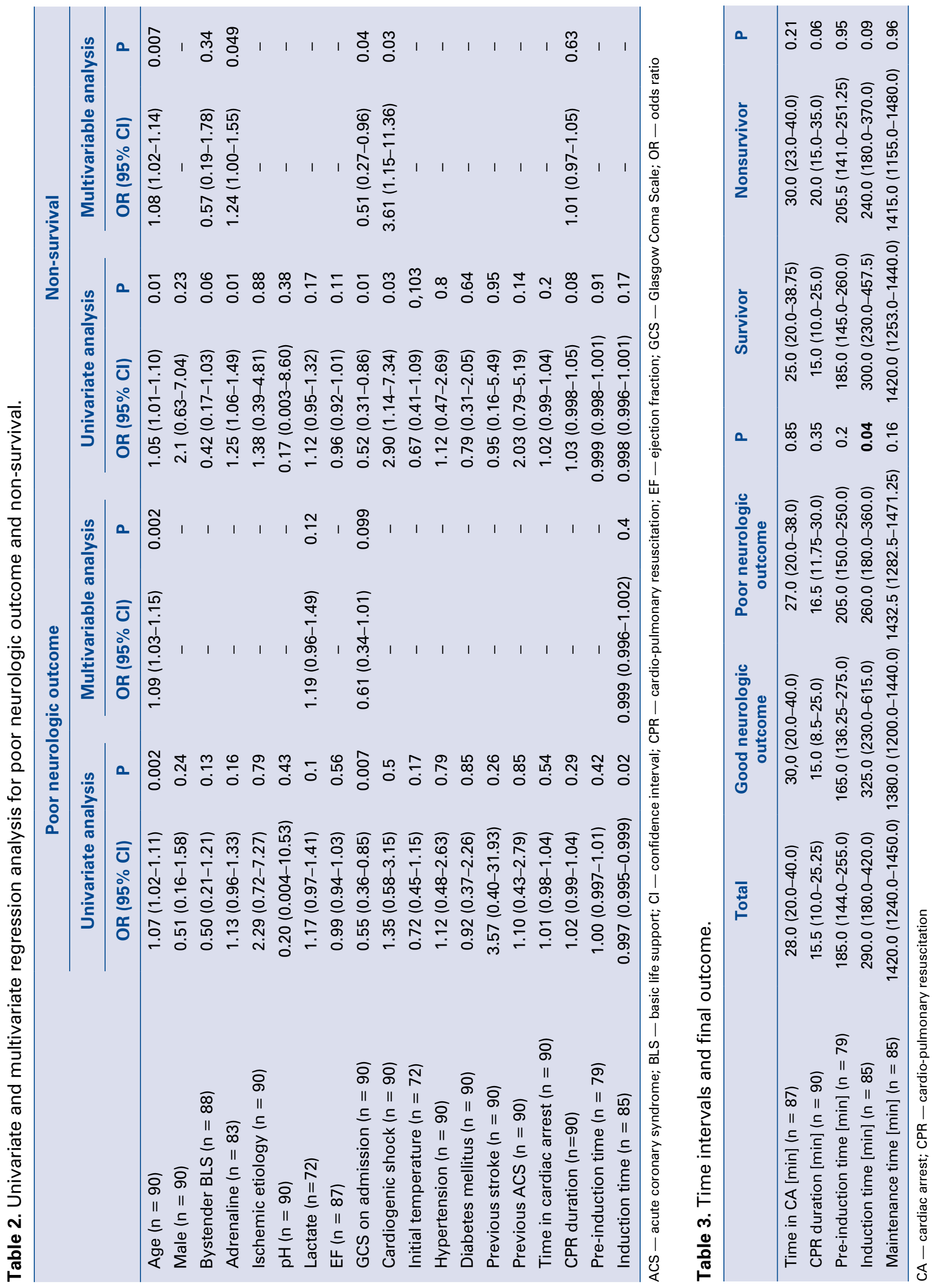
Table 4. Univariate and multivariate regression analysis for mild therapeutic hypothermia (MTH) induction time.

\begin{tabular}{|c|c|c|c|c|}
\hline & \multicolumn{2}{|c|}{ Univariate analysis } & \multicolumn{2}{|c|}{ Multivariable analysis } \\
\hline & $\begin{array}{l}\text { Unstandardised } \\
\text { coefficient }(95 \% \mathrm{CI})\end{array}$ & $\mathbf{P}$ & $\begin{array}{l}\text { Unstandardised } \\
\text { coefficient }(95 \% \mathrm{CI})\end{array}$ & $\mathbf{P}$ \\
\hline Age $(n=90)$ & $-2.49(-6.39$ to 1.42$)$ & 0.21 & - & - \\
\hline Male $(n=90)$ & $-7.65(-129.93$ to 114.63$)$ & 0.9 & - & - \\
\hline Initial shockable rhythm $(n=90)$ & $81,27(-69.47$ to 232,0$)$ & 0.29 & - & - \\
\hline Bystender BLS ( $\mathrm{n}=88$ ) & $31.92(-67.54$ to 131.38$)$ & 0.53 & - & - \\
\hline Adrenaline $(n=83)$ & $-9.362(-26.98$ to 8.26$)$ & 0.29 & - & - \\
\hline Ischemic etiology $(n=90)$ & $-13.97(-148.9$ to 120.967$)$ & 0.62 & - & - \\
\hline $\mathrm{pH}(\mathrm{n}=90)$ & 124.76 (-319.6 to 569.13 ) & 0.58 & - & - \\
\hline Lactate $(n=72)$ & $-20.38(-38.56$ to -2.2$)$ & 0.03 & $-16.2(-34.49$ to 2.1$)$ & 0.08 \\
\hline$E F(n=87)$ & $0.964(-4.307$ to 6.235$)$ & 0.72 & - & - \\
\hline GCS on admission $(n=90)$ & $47.87(1.63$ to 94.11$)$ & 0.04 & $25.77(-27.31$ to 78.87$)$ & 0.34 \\
\hline Cardiogenic shock $(\mathrm{n}=90)$ & $-40.06(-137.89-57.78)$ & 0.42 & - & - \\
\hline Initial temperature $(n=72)$ & 116.75 (73.17 to 160.33 ) & $<0.001$ & - & - \\
\hline Hypertension $(\mathrm{n}=90)$ & $52.69(-45.36$ to 150.73$)$ & 0.29 & - & - \\
\hline Diabetes mellitus ( $n=90$ ) & $85.84(-17.14$ to 188.82$)$ & 0.101 & - & - \\
\hline Previous stroke $(n=90)$ & $-116.04(-310.5$ to 78.43$)$ & 0.24 & - & - \\
\hline Previous ACS $(n=90)$ & $47.31(-60.12$ to 78.43$)$ & 0.38 & - & - \\
\hline Time in cardiac arrest $(n=90)$ & $-3.35(-6.25$ to -0.45$)$ & 0.02 & $3.31(-4.48$ to 11.01$)$ & 0.4 \\
\hline CPR duration $(n=90)$ & $-4.23(-7.05$ to -1.4$)$ & 0.004 & $-6.75(-14.62$ to 1.11$)$ & 0.09 \\
\hline Pre-induction time $(\mathrm{n}=79)$ & $0.17(-0.42$ to 0.762$)$ & 0.56 & - & - \\
\hline
\end{tabular}

ACS - acute coronary syndrome; BLS — basic life support; $\mathrm{Cl}$ - confidence interval; CPR — cardio-pulmonary resuscitation; EF — ejection fraction; GCS - Glasgow Coma Scale

MTH [25] were associated with better neurologic outcome. Lee et al. [15] analyzed 515 patients treated with MTH in terms of time intervals and neurologic outcome. Despite a significant difference in the induction time between the groups with favorable and unfavorable outcome and no difference in the pre-induction time, regression analysis revealed the opposite results. No association between induction time and outcome were found while elongation of pre-induction by each 30 min increased the odds for poor outcome by $11 \%$. In the present study, induction time was also found to be significantly longer in patients with good neurologic outcome when compared with the poor outcome group. Subsequent univariate analysis revealed an association between MTH induction time and neurological outcome, however, similar to the results presented by Lee et al. [15], the finding was not confirmed in the multivariate model. Unlike the analysis by Haugk et al. [13] and Perman et al. [14], the induction time was not categorized to avoid a possible bias resulting from switching from a continuous to categorical variable. In the current study, older age and lower GCS score on admission were the only independent risk factors of poor neurologic outcome, these observations are consistent with previous studies $[15,19]$. The phenomenon of shorter induction time in patients with unfavorable outcome was suspected to be a result of more severe initial brain injury, impaired thermoregulation, and greater vulnerability to cooling [14]. A study evaluating heat generation in patients treated with MTH after cardiac arrest revealed an association between greater heat production and better baseline health status, reduced ischemic injury and improved neurologic outcome [26]. A study published by Leão et al. [27] showed that, apart from shorter induction time, patients with unfavorable neurologic outcome had a higher incidence of hypoxic-ischemic brain injury on magnetic resonance imagining and a higher concentration of neuron specific enolase. The present results are consistent with these studies $[14,15,19,26,27]$ and might indirectly support the 
hypothesis that shorter induction time in patients with unfavorable neurological outcome is related to more severe brain injury, since the initial neurologic condition reflected by GCS on admission was a strong outcome predictor. Furthermore, an association between longer MTH induction time and higher GCS score was found in univariate analysis, however results were not confirmed in the multivariate model. The differences between the studies regarding time intervals could result from the implementation of various definitions, different methods of cooling [28] and temperature measurement [29], and heterogeneity of the study populations (inclusion of patients regardless of the etiology and place of cardiac arrest or the type of the initial rhythm).

There were no differences in time intervals between survivors and non-survivors in the present study. Similar results were presented by Haugk et al. [13]. The current study found that older age, higher doses of adrenaline during CPR, lower GCS score on admission and presence of cardiogenic shock were predictors of in-hospital mortality. Lee et al. [15] also reported lower initial GCS scores along with nonshockable rhythm, longer time in cardiac arrest and a higher Sequential Organ Failure Assessment (SOFA) score were independent risk factors for mortality, while no association was found between other time variables (pre-induction and induction) and odds for survival. In contrast to previous results, Leão et al. [27] showed a correlation between higher mortality at 6 months after cardiac arrest and shorter time to TT.

Addressing the multiple inconsistencies regarding time intervals in TTM and particularly the MTH induction time, the predictors were determined for delayed achievement of TT. In univariate regression analysis, longer MTH induction time was associated with lower lactate level, higher GCS score on admission, shorter time in cardiac arrest, and shorter CPR duration. According to the multivariate analysis, only shorter CPR duration and lower lactate level on admission were associated with prolonged induction time. The role of initial temperature in patients treated with MTH was raised in some previous studies, linking lower initial temperature with in-hospital [30] and longterm [31] mortality. This parameter however, was excluded from analysis as a potential confounder.

\section{Limitations of the study}

One of the main limitations of the present study is its partly retrospective and observational nature. Furthermore, only a relatively small group of patients were analyzed. Another limitation was the difficulty in precisely determining all essential time points.

\section{Conclusions}

Favorable neurologic outcome (CPC 1-2) in OHCA patients treated with MTH is associated with younger age and higher GCS score upon admission. The risk factors for increased in-hospital mortality in this population included older age, higher doses of adrenaline during CPR, lower GCS score on admission and presence of cardiogenic shock. Neither the induction nor pre-induction time was an independent risk factor for neurologic outcome or overall survival. CPR duration and lactate level on admission were predictors for prolonged induction time.

\section{Acknowledgements}

This study has been partly developed as a substudy of the 'Diamentowy Grant' project financed by the Ministry of Science and Higher Education of the Republic of Poland from research funds for the years 2015-2018 (DI2014009144). The funder had no role in study design, data collection and analysis, decision to publish, or preparation of the manuscript.

\section{Conflict of interest: None declared}

\section{References}

1. Nadolny K, Bujak K, Kucap M, et al. The Silesian Registry of Out-of-Hospital Cardiac Arrest: Study design and results of a three-month pilot study. Cardiol J. 2018 [Epub ahead of print], doi: 10.5603/CJ.a2018.0140, indexed in Pubmed: 30444257.

2. Atwood C, Eisenberg MS, Herlitz J, et al. Incidence of EMStreated out-of-hospital cardiac arrest in Europe. Resuscitation. 2005; 67(1): 75-80, doi: 10.1016/j.resuscitation.2005.03.021, indexed in Pubmed: 16199289.

3. Szczerbinski S, Ratajczak J, Lach P, et al. Epidemiology and chronobiology of out-of-hospital cardiac arrest in a subpopulation of southern Poland: A two-year observation. Cardiol J. 2018 [Epub ahead of print], doi: 10.5603/CJ.a2018.0025, indexed in Pubmed: 29611174.

4. Pusswald G, Fertl E, Faltl M, et al. Neurological rehabilitation of severely disabled cardiac arrest survivors. Part II. Life situation of patients and families after treatment. Resuscitation. 2000; 47(3): 241-248, indexed in Pubmed: 11114453.

5. Donnino MW, Andersen LW, Berg KM, et al. Temperature Management After Cardiac Arrest: An Advisory Statement by the Advanced Life Support Task Force of the International Liaison Committee on Resuscitation and the American Heart Association Emergency Cardiovascular Care Committee and the Council on Cardiopulmonary, Critical Care, Perioperative and Resuscitation. Circulation. 2015; 132(25): 2448-2456, doi: 10.1161/ CIR.0000000000000313, indexed in Pubmed: 26434495. 
6. Ibanez B, James S, Agewall S, et al. 2017 ESC Guidelines for the management of acute myocardial infarction in patients presenting with ST-segment elevation: The Task Force for the management of acute myocardial infarction in patients presenting with ST-segment elevation of the European Society of Cardiology (ESC). Eur Heart J. 2018; 39(2): 119-177, doi: 10.1093/eurheartj/ ehx393, indexed in Pubmed: 28886621.

7. Freund B, Kaplan PW. A review of the utility of a hypothermia protocol in cardiac arrests due to non-shockable rhythms. Cardiol J. 2017; 24(3): 324-333, doi: 10.5603/CJ.a2017.0016, indexed in Pubmed: 28150290.

8. Bernard SA, Gray TW, Buist MD, et al. Treatment of comatose survivors of out-of-hospital cardiac arrest with induced hypothermia. N Engl J Med. 2002; 346(8): 557-563, doi: 10.1056/ NEJMoa003289, indexed in Pubmed: 11856794.

9. Hypothermia after Cardiac Arrest Study Group. Mild therapeutic hypothermia to improve the neurologic outcome after cardiac arrest. N Engl J Med. 2002; 346(8): 549-556, doi: 10.1056/NEJMoa012689, indexed in Pubmed: 11856793.

10. Kozinski M, Pstragowski K, Kubica JM, et al. ACS networkbased implementation of therapeutic hypothermia for the treatment of comatose out-of-hospital cardiac arrest survivors improves clinical outcomes: the first European experience. Scand J Trauma Resusc Emerg Med. 2013; 21: 22, doi: 10.1186/17577241-21-22, indexed in Pubmed: 23531402.

11. Nielsen N, Wetterslev J, Cronberg T, et al. Targeted temperature management at $33^{\circ} \mathrm{C}$ versus $36^{\circ} \mathrm{C}$ after cardiac arrest. N Engl J Med. 2013; 369(23): 2197-2206, doi: 10.1056/nejmoa1310519.

12. Umińska J, Koziński M, Pstragowski K, et al. Platelet reactivity during mild therapeutic hypothermia in patients with acute myocardial infarction treated with ticagrelor: study protocol of a single-centre study. Med Res J. 2016; 1(4): 115-119, doi: 10.5603/ mrj.2016.0021.

13. Haugk M, Testori C, Sterz F, et al. Relationship between time to target temperature and outcome in patients treated with therapeutic hypothermia after cardiac arrest. Crit Care. 2011; 15(2): R101, doi: 10.1186/cc10116, indexed in Pubmed: 21439038.

14. Perman SM, Ellenberg JH, Grossestreuer AV, et al. Shorter time to target temperature is associated with poor neurologic outcome in post-arrest patients treated with targeted temperature management. Resuscitation. 2015; 88: 114-119, doi: 10.1016/j. resuscitation.2014.10.018, indexed in Pubmed: 25447429.

15. Lee BK, Jeung KW, Jung YH, et al. Relationship between timing of cooling and outcomes in adult comatose cardiac arrest patients treated with targeted temperature management. Resuscitation. 2017; 113: 135-141, doi: 10.1016/j.resuscitation.2016.12.002, indexed in Pubmed: 27987398.

16. Kubica J, Pstragowski K, Adamski P, et al. Mild therapeutic hypothermia for patients with acute coronary syndrome and cardiac arrest treated with percutaneous coronary intervention (UNICORN). The design and rationale for the prospective, observational, multicenter study. Med Res J. 2016; 1(1): 23-27, doi: 10.5603/mrj.2016.0004.

17. Perkins G, Jacobs I, Nadkarni V, et al. Cardiac Arrest and Cardiopulmonary Resuscitation Outcome Reports: Update of the Utstein Resuscitation Registry Templates for Out-of-Hospital Cardiac Arrest. Resuscitation. 2015; 96: 328-340, doi: 10.1016/j. resuscitation.2014.11.002.

18. Booth CM, Boone RH, Tomlinson G, et al. Is this patient dead, vegetative, or severely neurologically impaired? Assessing outcome for comatose survivors of cardiac arrest. JAMA. 2004;
291(7): 870-879, doi: 10.1001/jama.291.7.870, indexed in Pubmed: 14970067.

19. Nielsen N, Hovdenes J, Nilsson F, et al. Hypothermia Network. Outcome, timing and adverse events in therapeutic hypothermia after out-of-hospital cardiac arrest. Acta Anaesthesiol Scand. 2009; 53(7): 926-934, doi: 10.1111/j.1399-6576.2009.02021.x, indexed in Pubmed: 19549271.

20. Chiota NA, Freeman WD, Barrett K. Earlier Hypothermia Attainment is Associated with Improved Outcomes after Cardiac Arrest. J Vasc Interv Neurol. 2011; 4(1): 14-17, indexed in Pubmed: 22518262.

21. Sendelbach S, Hearst MO, Johnson PJo, et al. Effects of variation in temperature management on cerebral performance category scores in patients who received therapeutic hypothermia post cardiac arrest. Resuscitation. 2012; 83(7): 829-834, doi: 10.1016/j. resuscitation.2011.12.026, indexed in Pubmed: 22230942.

22. Wolff B, Machill K, Schumacher D, et al. Early achievement of mild therapeutic hypothermia and the neurologic outcome after cardiac arrest. Int J Cardiol. 2009; 133(2): 223-228, doi: 10.1016/j. ijcard.2007.12.039, indexed in Pubmed: 18353458.

23. Schock RB, Janata A, Peacock WF, et al. Time to Cooling Is Associated with Resuscitation Outcomes. Ther Hypothermia Temp Manag. 2016; 6(4): 208-217, doi: 10.1089/ther.2016.0026, indexed in Pubmed: 27906641.

24. Kim WY, Ahn S, Hong JS, et al. The impact of downtime on neurologic intact survival in patients with targeted temperature management after out-of-hospital cardiac arrest: National multicenter cohort study. Resuscitation. 2016; 105: 203-208, doi: 10.1016/j.resuscitation.2016.03.020, indexed in Pubmed: 27060537.

25. Italian Cooling Experience (ICE) Study Group. Early- versus lateinitiation of therapeutic hypothermia after cardiac arrest: preliminary observations from the experience of 17 Italian intensive care units. Resuscitation. 2012; 83(7): 823-828, doi: 10.1016/j. resuscitation.2011.12.002, indexed in Pubmed: 22155700.

26. Murnin MR, Sonder P, Janssens GN, et al. Determinants of heat generation in patients treated with therapeutic hypothermia following cardiac arrest. J Am Heart Assoc. 2014; 3(3): e000580, doi: 10.1161/JAHA.113.000580, indexed in Pubmed: 24780205.

27. Leão RN, Ávila P, Cavaco R, et al. Therapeutic hypothermia after cardiac arrest: outcome predictors. Rev Bras Ter Intensiva. 2015; 27(4): 322-332, doi: 10.5935/0103-507X.20150056, indexed in Pubmed: 26761469 .

28. Klimczuk T, Kubica J, Kasprzak M, et al. Łagodna hipotermia terapeutyczna po nagłym zatrzymaniu krążenia w przebiegu ostrego zespołu wieńcowego - doświadczenia $\mathrm{z}$ wdrażania metody. Folia Cardiologica. 2015; 10(1): 19-24, doi: 10.5603/fc.2015.0005.

29. Umińska JM, Buszko K, Ratajczak J, et al. Comparison of temperature measurements in esophagus and urinary bladder in comatose patients after cardiac arrest undergoing mild therapeutic hypothermia. Cardiol J. 2018 [Epub ahead of print], doi: 10.5603/ CJ.a2018.0115, indexed in Pubmed: 30246234.

30. Benz-Woerner J, Delodder F, Benz R, et al. Body temperature regulation and outcome after cardiac arrest and therapeutic hypothermia. Resuscitation. 2012; 83(3): 338-342, doi: 10.1016/j. resuscitation.2011.10.026, indexed in Pubmed: 22079947.

31. Hovdenes J, Røysland K, Nielsen N, et al. A low body temperature on arrival at hospital following out-of-hospital-cardiacarrest is associated with increased mortality in the TTM-study. Resuscitation. 2016; 107: 102-106, doi: 10.1016/j.resuscitation.2016.08.011, indexed in Pubmed: 27565034. 